\title{
MAGNETOOPTICAL STUDIES OF QUASI-TWO-DIMENSIONAL ELECTRON LAYER ON HgCdMnTe
}

\author{
E. Dudziak, J. Bożym and D. PRUChNiK \\ Institute of Physics, Technical University of Wroclaw \\ Wybrzeże Wyspiańskiego 27, 50-370 Wrocław, Poland
}

Photovoltaic magneto-spectroscopy was used for the first time as the interband probe in the study of the 2D electron gas on narrow-gap HgCdMnTe semiconductor. The strong circular dichroism was found which agrees with the phenomenological description of the optical properties of the 2D electron systems in quantized Hall regime. The model of coupled intersubband resonances and interband transitions was proposed as a qualit itive explanation.

PACS numbers: 78.20.Ls, 73.30.+y, 72.40.+w

1. Optical properties of 2D electron gas in quantized Hall regime. Phenomenological approach

In the phenomenological approach the photo-response of the bulk material to a circularly polarized wave can be described by the complex index of refraction $(\eta-\mathrm{i} \kappa)$

$$
\left(\eta_{ \pm}-\mathrm{i} \kappa_{ \pm}\right)^{2}=\mu \varepsilon[1-(4 \pi \mathrm{i} / \omega \varepsilon)] \sigma_{ \pm}^{(3 \mathrm{D})}
$$

where high frequency conductivity

$$
\sigma_{ \pm}^{(3 \mathrm{D})}=\sigma_{x x}^{(3 \mathrm{D})} \pm \mathrm{i} \sigma_{x y}^{(3 \mathrm{D})}
$$

Following Peng et al. [1] in the transition from 3D to 2DEG (2D electron gas) in quantized Hall regime we obtain

$$
\sigma^{(3 \mathrm{D})}=\sigma^{(2 \mathrm{D})} / d
$$

and $\sigma_{x x}=\sigma_{y y}=0, \sigma_{x y}=\nu e^{2} / h$, where the Landau filling factor $\nu$ is integer or a rational fraction, $d$ is finite thickness of the layer. Putting

$$
\omega_{0}=\frac{4 \pi e^{2}}{h d}
$$

we have .

$$
\left(\eta_{ \pm}-\mathrm{i} \kappa_{ \pm}\right)^{2}=\mu\left(\varepsilon \pm \nu \omega_{0} / \omega\right) .
$$

As a result for $\omega \gg \omega_{0}$ we have quantum Faraday rotation

$$
|\Theta| \approx 2 \pi \nu \alpha / \eta
$$


where $\alpha=e^{2} / h c$ is the fine structure constant and a strong circular dichroism

$$
\kappa_{+} \approx 0, \quad \kappa_{-}=\left(\nu \omega_{0} / \omega\right)^{1 / 2}
$$

for $\omega \ll \omega_{0}$. Quantum Faraday effect has been observed in $\mathrm{GaAs}-\mathrm{Al}_{x} \mathrm{Ga}_{1-x} \mathrm{As}$ heterostructures in the microwave region by Kuchar et al. [2] and Volkov et al. [3]. Recently many papers [4-6] were devoted to the optical properties of quasi-two-dimensional electron systems in GaAs by interband spectroscopy, mainly luminescence. It was motivated, for example, by the possible use of such spectroscopy in studies of the quantum Hall effect in a contactless way.

The narrow-gap semiconductors (NGS) to which belongs our $\mathrm{HgCdMnTe}$ material are the special case of 2DEG. The first quantum transport studies of 2DEG at natural grain boundaries in $\mathrm{HgCdMnTe}$ and $\mathrm{HgMnTe}$ have been reported by Grabecki et al. [7, 8]. In Refs. [9, 10] the spin-resonance of surface electrons on narrow-gap semiconductors has been shown. In this work we present the results of magnetooptical studies of 2DEG on $\mathrm{HgCdMnTe}$ performed for the first time by means of photovoltaic (PV) effect spectroscopy.

\section{Experiment}

$\mathrm{Hg}_{0.78} \mathrm{Cd}_{0.2} \mathrm{Mn}_{0.02}$ Te narrow-gap semiconductor $\left(E_{\mathrm{g}}=131 \mathrm{meV}\right.$ at $\left.10 \mathrm{~K}\right)$ used in experiment was the epitaxial layer grown by isothermal vapour phase epitaxy. The as-grown $\mathrm{HgCdMnTe}$ had hole concentration $\approx 5 \times 10^{17} \mathrm{~cm}^{-3}$ and Hall mobilities of about $10^{2} \mathrm{~cm}^{2} /(\mathrm{V} \mathrm{s})$ at $77 \mathrm{~K}$. Magnetooptical measurements in Faraday configuration were performed on 2DEG between metal (gold) and $p$-type HgCdMnTe semiconductor. The interface plane was perpendicular to the magnetic field and the incident light beam (see inset in Fig. 1). The experiment was performed at temperatures of $10 \mathrm{~K}$ in magnetic fields up to $6 \mathrm{~T}$. Left and right

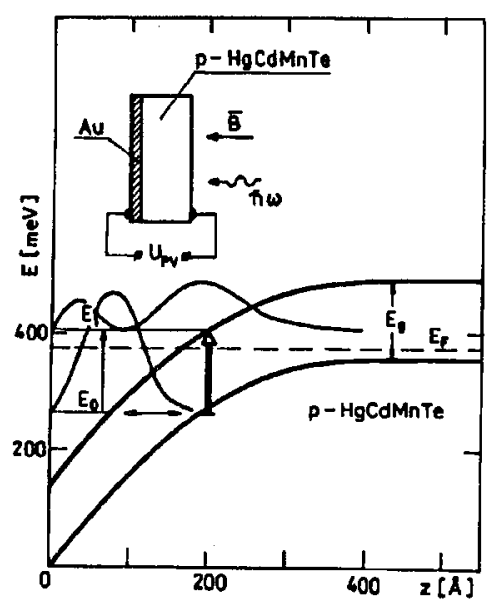

Fig. 1. Subband energies in the surface inversion layer on $p$-HgCdMnTe with $E_{g}=$ $131 \mathrm{meV}, N_{\mathrm{a}}=5 \times 10^{17} \mathrm{~cm}^{-3}$. The band bending is taken from [13] for $\mathrm{HgCdTe}$ with similar $E_{\mathbf{g}}$ and $N_{\mathrm{a}}$ parameters. The inset shows the experimental geometry. 
circularly polarized light was used. The photovoltaic spectra were recorded in a fixed magnetic field.

\section{Results and discussion}

We could not use photoluminescence spectroscopy as interband probes of 2DEG because there is no luminescence in very narrow-gap semiconductors. Instead we used PV effect as the measure of magnetoabsorption. The PV signal is obviously the result of interband transitions because the creation of PV voltage needs an excitation of electron-hole pairs and their separation in space by the potential barrier. The measured PV spectra at the different fixed magnetic fields for two circular polarizations are presented in Fig. 2a. The shape of the spectra differs from PV spectra of $p-n$ junctions in bulk systems of the NGS with similar values of energy gap [11]. The spectra of the 2DEG are much deeper modulated and their shape is close to the Fano lineshape [12]. The spectra are characterised by high signal to noise ratio. But the most striking feature of the spectra is the strong circular dichroism. We have never observed such strong dichroism in PV spectra in the bulk NGS. We interpret our results in the similar way as the au-
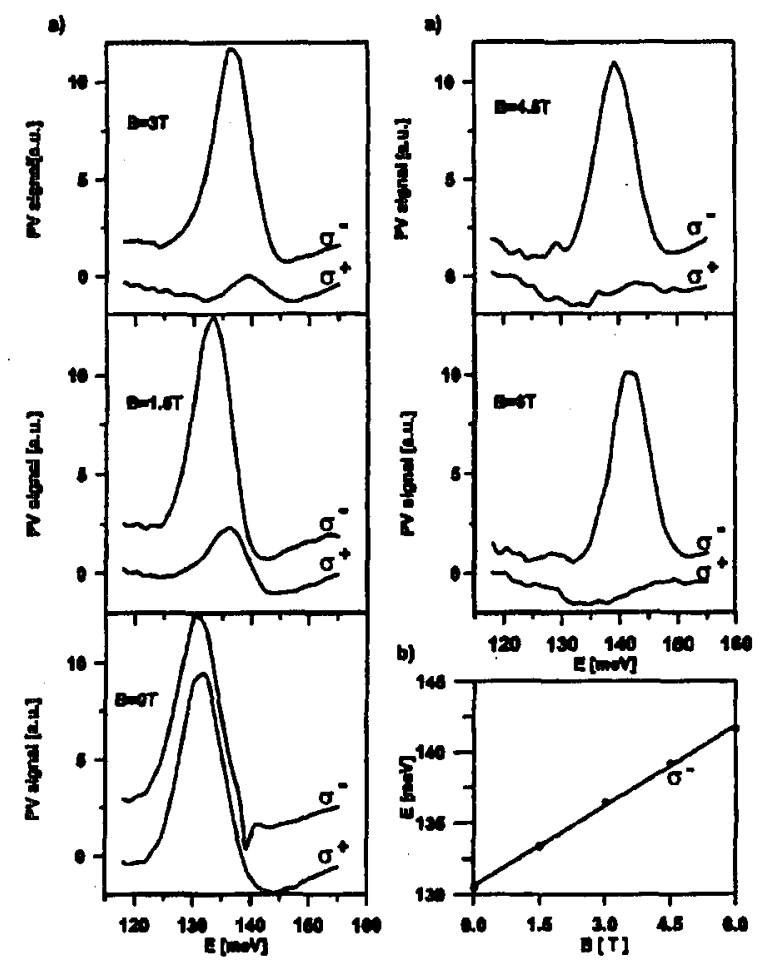

Fig. 2. (a) Photovoltaic spectra of 2DEG on $\mathrm{HgCdMnTe}$ in different magnetic fields for two circular $\sigma^{+}$and $\sigma^{-}$light polarizations. The spectra for $\sigma^{+}$are for clarity shifted down by 2 a.u. (b) Peak energies for $\sigma^{-}$polarization vs. magnetic field. 
thors of paper [13] in the case of interband-quantum-electroreflection $\Delta R / R$ from $p-\mathrm{HgCdTe}$ (without magnetic field). We think that the big peak in our PV spectra originates from transitions from the continuum of valence states into the discrete $E_{1}$ Landau state in the surface layer (Fig. 1). In a highly $p$-doped NGS the distance $E_{01}$ between the discrete subbands in 2DEG layer is comparable with the gap $E_{\mathrm{g}}$. The ground-state subband $E_{0}$ is degenerated with the valence band and there is tunneling interaction between them. The ground state $E_{0}$ perturbed by coupling with valence band (shifted in energy and broadened) is excited in intersubband resonance to a non-interacting discrete state $E_{1}$. This intersubband resonance interferes coherently with simultaneous transition from the valence continuum into $E_{1}$ level. The two contributions must be added phase-coherently [12] and then squared to obtain the effective absorption with characteristic Fano lineshape. One contribution of our process (intersubband resonance) depends on circular polarization state of the light. This involves the same polarization dependence of the second coupled transition - interband transition. From the slope of the linear peak energies dependence on magnetic field (Fig. 2b) reduced effective mass $m_{\mathrm{r}}^{*}=0.032 m_{\mathrm{e}}$ has been determined.

\section{References}

[1] Jian-Ping Peng, Shi-Xun Zhou, Xue-Chu Shen, Phys. Rev. B 44, 4021 (1991-II).

[2] F. Kuchar, R. Meisels, G. Weinmann, W. Schlapp, Phys. Rev. B 33, 2965 (1986).

[3] V.A. Volkov, D.A. Galchenkhov, L.A. Galchenkhov, I.M. Grodnenskij, O.R. Metov, S.A. Mikhailov, A.P. Senichkin, K.V. Starostin, Zh. Eksp. Teor. Fiz. Pisma Red. 43, 255 (1986).

[4] A.J. Turberfield, S.R. Haynes, P.A. Wright, R.A. Ford, R.G. Clark, J.F. Ryan, J.J. Harris, C.T. Foxon, Phys. Rev. Lett. 65, 637 (1990).

[5] B.B. Goldberg, D. Heiman, Phys. Rev. Lett. 65, 641 (1990).

[6] H. Buhmann, W. Joss, K. von Klitzing, I.V. Kukushkin, G. Martinez, A.S. Plant, K. Ploog, V.B. Timofeev, Phys. Rev. Lett. 65, 1056 (1990).

[7] G. Grabecki, T. Dietl, P. Sobkowicz, J. Kossut, W. Zawadzki, Appl. Phys. Lett. 45, 11 (1984).

[8] G. Grabecki, A. Wittlin, T. Dietl, P.A.A. Teunissen, S.A.J. Wiegers, J.A.A.J. Perenboom, Semicond. Sci. Techrol. 8, S95 (1993).

[9] M. Chmielowski, T. Dietl, P. Sobkowicz, F. Koch, 18th Int. Conf. Physics of Semicond., Stockholm 1986, Ed. O. Engstrom, Vol. 2, World Scientific, Singapore 1987, p. 1787.

[10] R. Sizmann, Junkao Chu, R. Wollrab, F. Koch, J. Ziegler, H. Maier, 19th Int. Conf. Physics of Semicond., Warsaw 1986, Ed. W. Zawadzki, Vol. 1, Institute of Physics PAS, Warsaw 1988, p. 471.

[11] W. Zawadzki, E. Dudziak, L.Z. Jędral, E. Płaczek-Popko, J. Bożym, Semicond. Sci. Technol. 8, S172 (1993).

[12] U. Fano, Phys. Rev. 124, 1866 (1961).

[13] R. Sizmann, M. Zass, F. Koch, Surf. Sci. 229, 444 (1990). 